\title{
Orientation Adjustment of Microscale Particles for Quantitative SEM-EDS Analysis
}

\author{
Chunfei $\mathrm{Li}^{1}$, Alexander Huey ${ }^{2}$, Jeremy Marshall ${ }^{1}$ and Coleman Milligan ${ }^{1}$ \\ ${ }^{1}$ Clarion University, Clarion, Pennsylvania, United States, ${ }^{2}$ California University of Pennsylvania, United \\ States
}

Quantitative SEM-EDS analysis requires that the specimen have a flat surface and the flat surface is perpendicular to the electron beam [1]. This condition is not satisfied automatically for the analysis of small particles, since the particles are typically randomly oriented inside SEM and there is no accepted procedure for in-situ orientation adjustment [2]. Since the specimen stage of typical SEM is equipped with rotation and tilting function as shown in Fig. 1 (a), it is possible to perform an in-situ specimen orientation adjustment to satisfy the perpendicular requirement. This paper suggests a procedure for such adjustment and reports the preliminary result. The layout of a microscale specimen on a SEM stage can be represented by the schematic drawing shown in Fig. 1(a). The top flat surface of the specimen is inclined relative to the top-surface of the stage. For the convenience of explanation, we assume the specimen is wedge-shaped. It is further assumed that the electron beam comes straight down. Therefore, the goal of orientation adjustment is to make the top surface of the wedge parallel to horizontal. The top-surface of the wedge intercepts with the top of the stage, forming an interception line. The goal can be achieved: first by using rotation function to bring the interception line parallel to the tilt axis as shown in Fig. 1(b); then, by manipulating the tilt function only, as shown in Figs. 1(b)-(c). The challenge is that inside SEM, the specimen is of irregular shape and there is no physical interception line visible. The tools available to us are that we know the relative orientation of the rotation and tilting axis's to the SEM image and we can take multiple SEM images at different stage rotation and tilt angles. The following procedure has been proposed. First, we find the stage rotation angle at which the (virtual) interception line is parallel to the stage-tilting axis through the following operations. Take a SEM image at 00 tilt angle. On the image, choose two features represented by A' and B'. Make sure line A'B' is perpendicular to the tilting axis of the stage. Measure the distance from A' to B'. Tilt the stage from 00 to 100 while retain the stage rotation angle. Take another SEM image and measure the distance A'B' again. Calculate the difference of distance A'B' and calculate the ratio of distance difference to the original value. Change the stage rotation angles, for example by 100 , and repeat the above steps. Plot the ratio as a function of rotation angle. A simulated plot is shown in Fig. 2(c), where 1800 rotation angle corresponds to the case when the interception line is parallel to the stage tilting axis by design. As seen in Fig. 2(c), this special rotation angle corresponds to a local minimum on the plot. Therefore, on a similar graph generated by using experimental data, we can find the special rotation angle by looking for the local minimum. The reason for the unique shape of the plot is briefly explained here. As shown in Fig. 2(b), A and B are two chosen points on the surface of the specimen and the line connecting them is perpendicular to the tilt axis at a specific rotation angle. A' and B' are the projection of the two points onto horizontal. Distance A'B' is a function of the tilt angle of line AB relative to the horizontal, which, in turn, is a function of stage rotation angles. Therefore, the ratio changes with stage rotation angle and is expected to have a special value when the interception line is parallel to the tilt axis. The special value turned out to be a local minimum in the case of simulation. Once the stage is set at this special rotation angle, the top surface can be brought to horizontal through manipulating the tilting function only as shown in Figs. 1(b) and (c). In this case, distance A'B' changes with the stage tilt angle, reaching a maximum at some point. At this special tilt angle, the specimen surface is parallel to the horizontal. A preliminary experiment along this line has been carried out inside SEM with microscale specimen. While the experimental results resemble the simulated one, the two differ in many details. Therefore, it is determined that this procedure has to be tested outside SEM with macroscale sample, where the effectiveness can be confirmed and potential problems can be identified straightforward. For this purpose, a stage functions the same as one 
for SEM but can be used outside SEM has been built. A wedge-shaped object of ten-centimeter size is placed on top of the stage to represent the specimen. The key of the experiment is the measurement of distance A'B', for which two methods has been proposed. In the first method, two points A and B satisfying the conditions explained above will be marked on the surface of the macroscale specimen at certain stage rotation angle. Two vertical threads passing through points A and B will be produced. The distance between the threads is A'B' and will be measured directly. The second one is based on a photograph of top-down view . The distance will be measured from the image. The first approach of direct measurement is closer to the theoretical consideration while that of photo measurement is closer to what happens in SEM. Comparing the result of the two methods allows us to examine any unexpected discrepancy between hypothesis and the result of practical operation and to find a solution promptly. Data generated by using the first method has shown promising result.

Acknowledgement Financial support by National Science Foundation (DMR-1900077) is acknowledged.
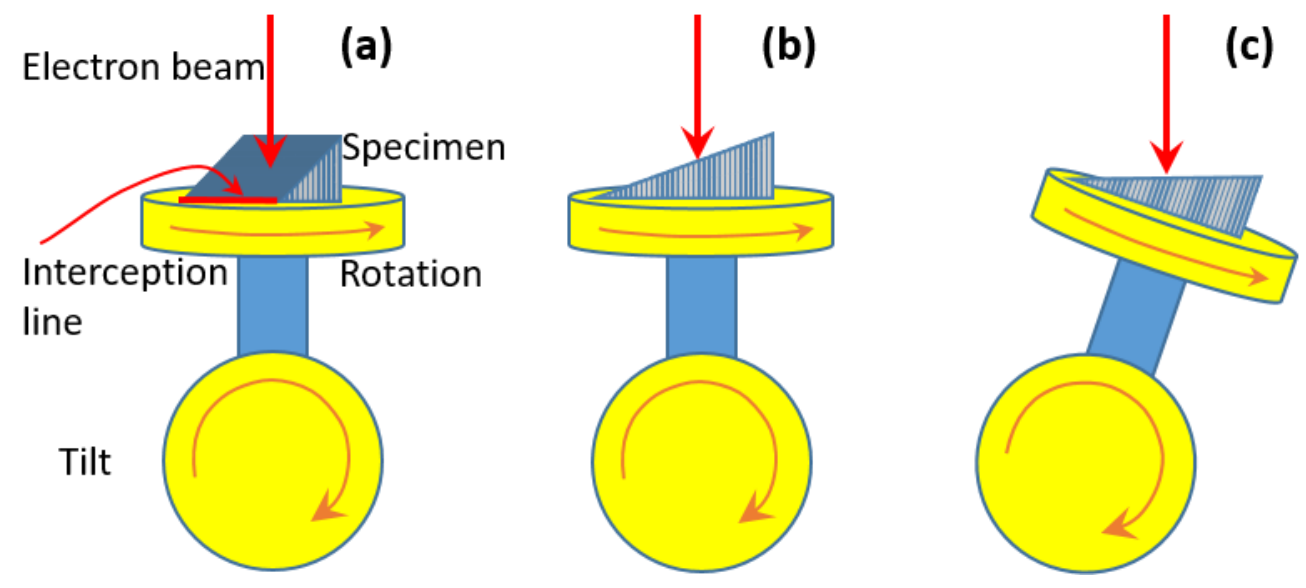

Figure 1. Schematic drawing illustrating the procedure to bring an inclined surface to horizontal with the rotation and tilting functions of a SEM stage. From (a) to (b), the interception line is made parallel to the tilt axis by manipulating the rotation function. From (b) to (c), the top surface of the specimen is adjusted to be parallel to the horizontal by using tilting function. 

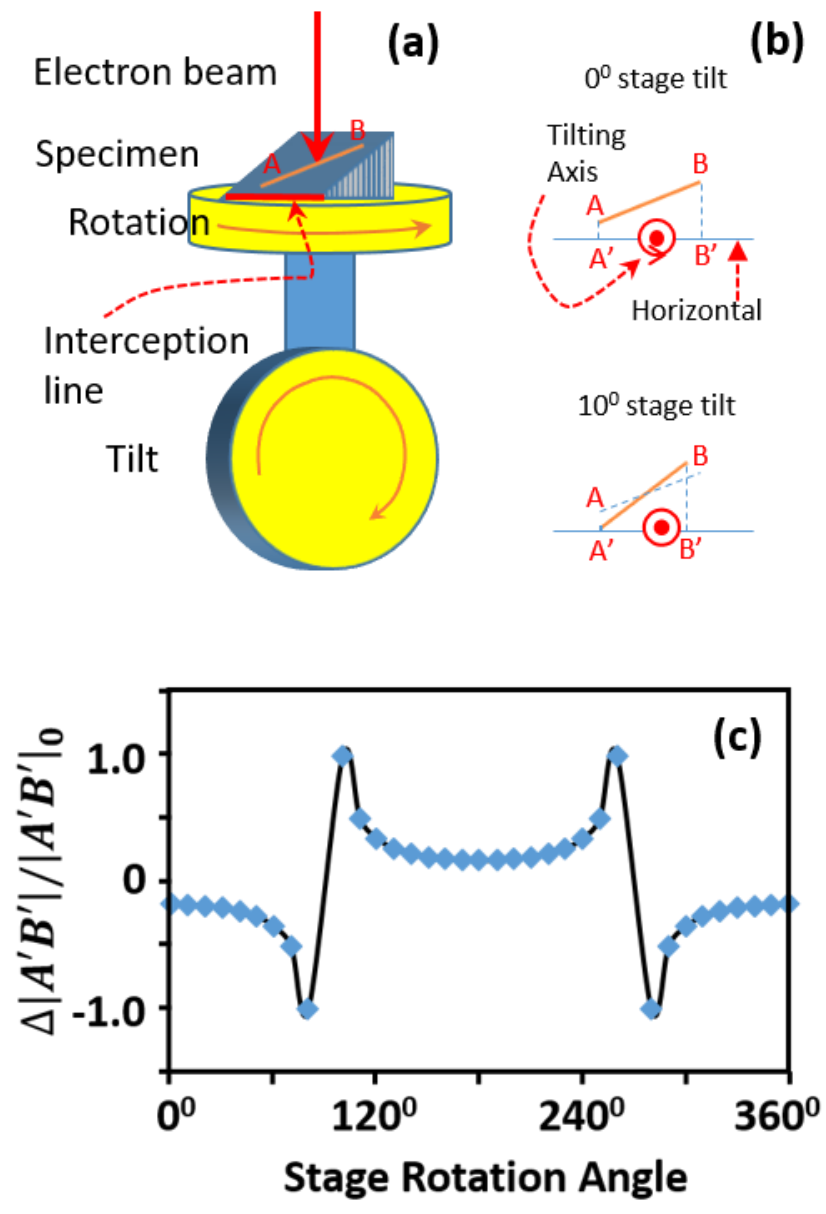

Figure 2. Schematic drawing (a) and (b) illustrate the relationship between the distances measured on images and the real value on specimen. (c) is a result of simulation showing the expected shape for the graph of the ratio as a function of stage rotation angle.

References

1. Goldstein, J. I., Newbury, D. E., Michael, J. R., Ritchie, N. W., Scott, J. H. J., \& Joy, D. C. (2017). Scanning electron microscopy and X-ray microanalysis. Springer.

2. Stevens, K. A. (1983). Surface Tilt(the direction of slant): A Neglected Psychophysical Variable (Master's thesis, Massachusetts Institute of Technology, 1983), Cambridge.

3. Duley, Niespodzianski, Merando, J. Marshall, and C. Li, EDS Analysis of Icosahedral Quasicrystalline Thin Film in Al65Cu25Fe15 Alloy Prepared by Arc-melting. Microscopy and Microanalysis, 1-3 (2020). doi:10.1017/S1431927620020735. 